Acta Crystallographica Section A

Foundations of Crystallography

ISSN 0108-7673

Received 1 March 2004

Accepted 18 June 2004

(C) 2004 International Union of Crystallography Printed in Great Britain - all rights reserved

\section{On the role of data quality in experimental charge- density studies}

\author{
Riccardo Destro, ${ }^{\text {a* }}$ Laura Loconte, ${ }^{\mathrm{a}}$ Leonardo Lo Presti, ${ }^{\mathrm{a}}$ Pietro Roversi ${ }^{\mathrm{b}}$ and \\ Raffaella Soave $^{c}$
}

a Department of Physical Chemistry and Electrochemistry, University of Milan, Via Golgi 19, 20133 Milano, Italy, ${ }^{\mathbf{b}}$ Department of Biochemistry, Laboratory of Molecular Biophysics, University of Oxford, South Parks Road, Oxford OX1 3QU, England, and ' CNR-ISTM, Via Golgi 19, 20133 Milano, Italy. Correspondence e-mail: riccardo.destro@unimi.it

\begin{abstract}
High-resolution X-ray diffraction data were collected at $T=19 \mathrm{~K}$ from two similar spherical crystals of the fungal metabolite citrinin, $\mathrm{C}_{13} \mathrm{H}_{14} \mathrm{O}_{5}$. The two data sets were of markedly different quality, one data set (MQ, medium quality) comprising a single octant of the reciprocal lattice and including reflections with obviously peculiar intensity profiles while the second (HQ, high quality) comprised a hemisphere of reflections and showed no flawed profiles. Parallel multipolar refinements were carried out for both. While most of the resulting geometric parameters, including bond lengths and angles, were in close agreement (the standard uncertainties were approximately twice as large for the MQ data, reflecting the smaller number of observations), the agreement is noticeably worse for electronic properties such as electron densities and their Laplacians at the bond and ring critical points. These latter features are especially sensitive to the quality of the low-angle (and strong) intensities, which was not high for the MQ data. By contrast, the magnitudes of the molecular dipole moment from the two experiments are the same within 1 standard uncertainty, with an angle of about $13^{\circ}$ between the two vectors. It is concluded that only true high-quality data allow a fully significant and quantitative analysis of the details of the experimental electron density $\rho_{\text {exp }}$, while high-resolution medium-quality data, measured at very low temperature and adequately processed, can still be used for a qualitative analysis, or for the derivation of overall electronic properties.
\end{abstract}

\section{Introduction}

A firm tenet in the field of X-ray charge-density research is the necessity of high-quality diffraction data for meaningful investigations of the experimental electron-density distributions $\left(\rho_{\text {exp }}\right)$ in crystals (Coppens \& Becker, 1995). Therefore, it is continuous practice to examine carefully the various factors that make up an optimal data set, as recently done, for example, in the case of the usage of CCD detectors (Sørensen \& Larsen, 2003, and references therein).

Much less investigated, or perhaps never explicitly addressed, is the influence of data quality on the results of an experimental charge-density study. To the question: 'what do we really gain in using high-quality data?', the obvious answer is 'accuracy and precision', but how relevant are accuracy and precision in this field, besides being always highly desirable? In general, X-ray charge-density research aims to extract chemical and physical information from a detailed analysis of the crystal electron distribution $\rho_{\exp }$. There is a certain dependence of the final numerical results on the model adopted to interpret the X-ray diffraction experiment, and in our opinion this dependence should be further investigated, to assess which models are the more adequate. A basis for such an assessment could be, when possible and appropriate, a comparison of the same physical quantities as determined by $\mathrm{X}$-ray diffraction with those measured by different techniques or calculated by periodic ab initio methods. But the obvious prerequisite is that the X-ray data be reliable, to avoid experimental deficiency being interpreted as more or less unexpected features of $\rho_{\exp }$.

To learn from a real case how severe the influence of data quality might be on an X-ray electron-density analysis, we have compared the outcomes of two experiments on crystals of the same compound. High-resolution low-temperature data have been collected in both experiments; to ensure a proper comparison, data processing and analysis, with multipole refinement, followed exactly the same procedures in both cases. The differences between the two experiments are in the crystal quality (affecting the accuracy) and in the extent of redundancy in data collection (hence in precision). The less accurate and less precise experiment led to data that we judge of medium, if not modest, quality (MQ hereinafter), the other 
gave a final set of high-quality (HQ) data. The main focus of the comparison will be on the topological properties of $\rho_{\text {exp }}$, but conventional (positional and thermal) parameters as well as molecular electric moments will also be examined.

\section{Experimental}

The crystals of the fungal metabolite citrinin (schematic drawing shown in Fig. 1) have long been investigated in our laboratory. The original interest was in the solid-state tautomeric equilibrium between the so-called para-quinonemethide form (Fig. 1) and the ortho form, obtained from the $p$-form by the transfer of two protons in rings III and IV from the $-\mathrm{OH}$ groups to the contiguous $-\mathrm{C}=\mathrm{O}$ groups, with a consequent rearrangement of all three double bonds of the two rings. X-ray measurements at 290 and 147 K (Destro \& Marsh, 1984) proved that at room temperature (RT) the crystals of citrinin are a tautomeric mixture of the $p$ - and $o$-forms in a ratio of about 3:2, while at the lower temperature only the $p$ tautomer is present in the crystals. Subsequent investigations in the

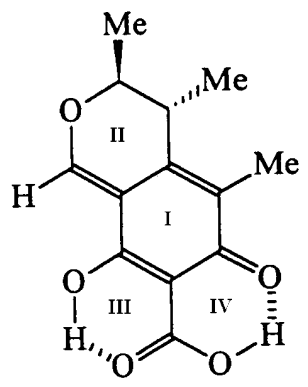

Figure 1

Molecular scheme of citrinin with ring numbering.
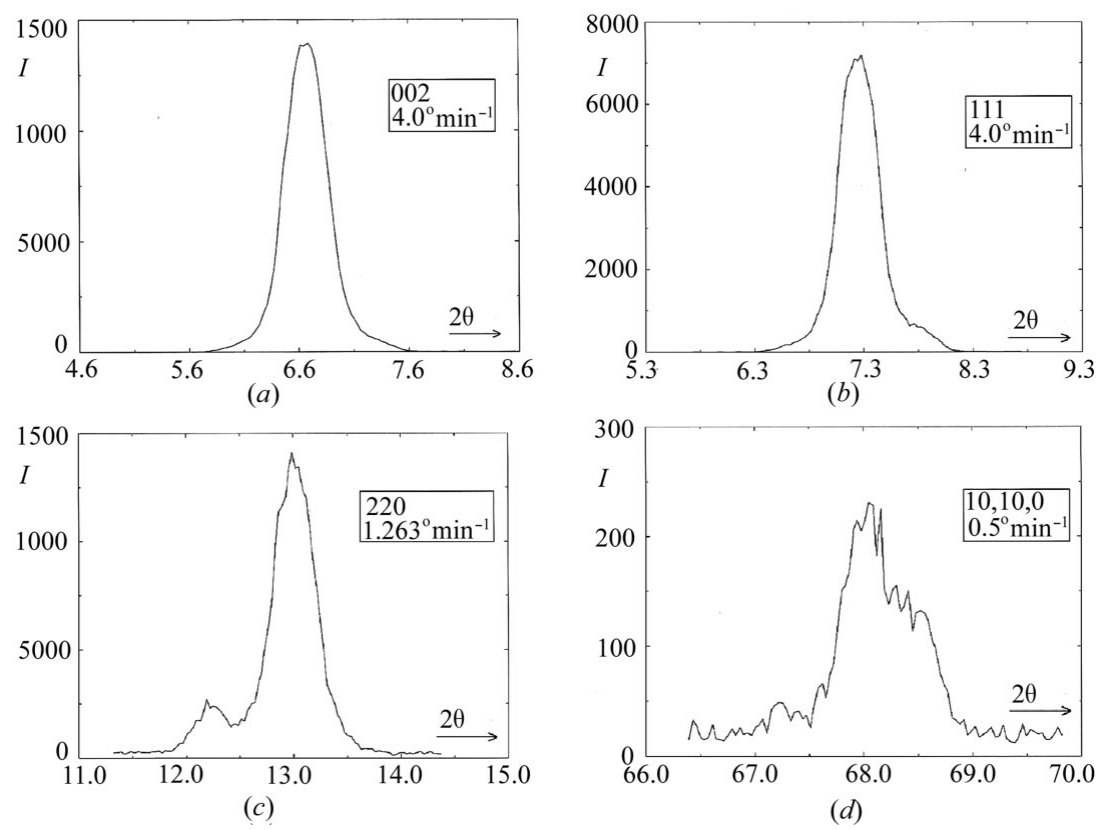

Figure 2

Some reflection profiles at $19 \mathrm{~K}$ from the medium-quality (MQ) experiment. Intensity (counts $\mathrm{s}^{-1}$ ) versus $2 \theta\left(^{\circ}\right)$ is reported, with reflection indices and scan rate in the inset boxes.
Table 1

Low-temperature (LT) crystal data and data collections for citrinin.

Chemical formula $\mathrm{C}_{13} \mathrm{H}_{14} \mathrm{O}_{5}, \mathrm{FW}=250.25, Z=4$, orthorhombic, space group $P 2_{1} 2_{1} 2_{1}, F(000)=528$.

\begin{tabular}{lll}
\hline Crystal & MQ & HQ \\
\hline Temperature $(\mathrm{K})$ & $19.5(10)$ & $19.0(10)$ \\
Diffractometer & Caltech $P \overline{1}$ & Milan $P \overline{1}$ \\
Crystal form & Sphere & Sphere \\
Crystal radius (mm) & 0.19 & 0.18 \\
Radiation type & Mo $K \alpha$ & Mo $K \alpha$ \\
$\lambda(\AA)$ & 0.71073 & 0.71073 \\
Unit-cell dimensions & & \\
$\quad a(\AA)$ & $13.251(4)$ & $13.255(1)$ \\
$\quad b(\AA)$ & $7.235(2)$ & $7.2377(7)$ \\
$\quad c(\AA)$ & $12.135(3)$ & $12.136(2)$ \\
$\quad V\left(\AA^{3}\right)$ & $1163.4(5)$ & $1164.3(3)$ \\
Data collection & & \\
(sin $\theta / \lambda)_{\text {max }}\left(\AA^{-1}\right)$ & 1.142 & 1.141 \\
Measured reflections & 8894 & 36564 \\
Independent reflections & 7958 & 7954 \\
Observed $(I>0)$ reflections & 7469 & 7698 \\
Scan rate $(2 \theta)\left({ }^{\circ}\right.$ min $\left.^{-1}\right)$ & $2.0-6.0$ & 3.0 \\
\hline
\end{tabular}

temperature range $20-293 \mathrm{~K}$ allowed us to fully characterize the equilibrium thermodynamics: enthalpy and entropy values, first derived from X-ray data alone (Destro, 1991), were later confirmed by solid-state ${ }^{13} \mathrm{C}$ NMR measurements (Poupko et al., 1997).

Our other works on crystalline citrinin were charge-density analyses. A preliminary account (Destro \& Merati, 1993) was followed some years later by a full report (Roversi et al., 1996), hereinafter referred to as paper I. The results in both papers were based on the data set that we classify here as HQ (see Table 1) but corrections for scan-truncation errors (Destro, 1988; Destro \& Marsh, 1987, 1993) had not been applied at the preliminary account stage.

Actually, other X-ray intensities, diffracted from a similar crystal, had been measured earlier (1986) at the California Institute of Technology (Caltech) in Pasadena (USA), at $T=19.5 \mathrm{~K}$ and up to $(\sin \theta / \lambda)=1.155 \AA^{-1}$ (that is, $2 \theta_{\mathrm{Mo}}=110.35^{\circ}$ ). The portion of them within $2 \theta_{\mathrm{Mo}}=108.5^{\circ}$ (for the sake of proper comparison with the HQ experiment) is reported in Table 1 as the MQ set. Indeed, the whole data set had been used and analysed (Destro \& Marsh, 1987) to estimate the scantruncation losses, but during this process some anomalous profiles were found, as shown in Fig. 2(c). Consequently, the data were judged of insufficient quality for a $\rho_{\exp }$ study. Extraneous peaks such as that found in the profile of reflection 220 (Fig. $2 c$ ) could be detected in 7 data out of the 1537 within the $\mathrm{Cu}$ sphere, while a larger portion of the profiles was affected by anomalous tails, as in the case of reflection 111 (Fig. 2b). Most of the data showed regular shapes in their profiles, as in Fig. 2(a) for reflection 002, and the double- 
Table 2

Room-temperature (RT) data collections and refinements.

\begin{tabular}{lllll}
\hline Crystal & MQ & & & HQ \\
\hline $\begin{array}{l}\text { Data collection } \\
\text { Diffractometer }\end{array}$ & $\begin{array}{c}\text { Caltech } \\
\overline{\text { 1 }}\end{array}$ & $\begin{array}{c}\text { Siemens } \\
\text { P4 }\end{array}$ & $\begin{array}{c}\text { Bruker Smart } \\
\text { APEX }\end{array}$ & $\begin{array}{c}\text { Milan } \\
P \overline{1}\end{array}$ \\
$\begin{array}{l}\text { Measured reflections } \\
\quad 3517\end{array}$ & 5951 & 11162 & 3455 \\
$\quad\left[\right.$ max $\left.\left(2 \theta_{\text {Mo }}\right)=55^{\circ}\right]$ & & & & \\
$R_{\text {int }}$ & 0.0237 & 0.0223 & 0.0211 & 0.0234 \\
$\begin{array}{l}\text { Independent } \\
\quad \text { reflections }\end{array}$ & 1612 & 1605 & 1622 & 1611 \\
$\begin{array}{l}\text { Refinement } \\
R(F)[I>2 \sigma(I)]\end{array}$ & & & & \\
$w R\left(F^{2}\right)$ & 0.0530 & 0.0520 & 0.0493 & 0.0418 \\
GoF & 0.1524 & 0.1399 & 0.1448 & 0.0743 \\
\hline
\end{tabular}

peak feature could be clearly detected only in low-angle data, but its presence could not be ruled out in some high-order reflections (Fig. 2d).

It is worth noting that crystal quality had been checked, prior to the low- $T$ data collection, by film techniques and some $\psi$ scans (a procedure that had led to the discard of a previous sample), but no anomalies were apparent. On the other hand, a more careful and closer inspection of the profiles in the full set of RT data, within $2 \theta_{\mathrm{Mo}}=55^{\circ}$, that we had also collected before the low- $T$ experiment, would have shown the same shape of Fig. 2(c) for reflection 220, as we realized a posteriori.

For the present work, we have checked if RT data measured with a CCD detector could have given some hints on the quality of the sample. To this purpose, the same 1986 crystal (now labelled MQ) was first mounted on a Siemens $P 4$ diffractometer (with a scintillation counter), to check if peak widths were still those of the Caltech 1986 measurements (to verify that no crystal degradation had occurred in the meanwhile), then on a Bruker Smart Apex diffractometer (see Table 2) equipped with a CCD detector. RT data collections on both instruments and their processing revealed no anomalies at a standard inspection. In other words, crystal quality would have been judged as normal, in the absence of a close, extensive and detailed profile analysis. A discussion on the relatively high values of $R(F)$ reported in Table 2 is postponed to a following section.

\section{Low-temperature (LT) data processing and multipolar refinements}

A careful and detailed report on the treatment of the HQ set of LT data, especially to correct them for scan truncation losses, has been given in paper I. The same procedure, including the evaluation of the 'true' background from weak reflections, has been applied to the MQ data for the present work. Raw LT diffraction intensities of both MQ and HQ samples were also processed according to the conventional protocol and the corresponding final sets are labelled in the following as 'no TR corr.' data.

Similarly, the same multipole model (Stewart, 1976) was adopted to interpret both HQ and MQ sets. It has been fully
Table 3

Summary of least-squares refinements A-D of the same multipolar model on MQ and HQ data.

For refinements $\mathrm{C}$ and $\mathrm{D}$, the second line reports the values of the statistical indices for data within $2 \theta_{\mathrm{Mo}}=55^{\circ}$ (Cu sphere).

\begin{tabular}{llllllll}
\hline & $\mathrm{MQ}$ & & & & $\mathrm{HQ}$ & & \\
\cline { 6 - 7 } & $R(F)$ & $w R\left(F^{2}\right)$ & $\mathrm{GoF}$ & & $R(F)$ & $w R\left(F^{2}\right)$ & $\mathrm{GoF}$ \\
\hline Refinement $\dagger$ & & & & & & \\
$\mathrm{A}$ & 0.0845 & 0.0812 & 0.9702 & & 0.0384 & 0.0382 & 1.3786 \\
$\mathrm{~B}$ & 0.0546 & 0.0730 & 1.0501 & & 0.0319 & 0.0371 & 1.4003 \\
$\mathrm{C}$ & 0.0664 & 0.0756 & 1.0193 & & 0.0281 & 0.0325 & 1.2267 \\
$\mathrm{D}$ & 0.0218 & 0.0400 & - & & 0.0090 & 0.0174 & - \\
& 0.0437 & 0.0675 & 1.0503 & & 0.0221 & 0.0310 & 1.2297 \\
& 0.0191 & 0.0390 & - & & 0.0080 & 0.0171 & -
\end{tabular}

$\dagger$ A: all observed $(I>0)$ data, no TR corr.; B: $I>2 \sigma(I)$, no TR corr.; C: all observed $(I>0)$ data, TR corr.; D: $I>2 \sigma(I)$, TR corr. The number of observed data in refinement $\mathrm{C}$, for both MQ and HQ crystals, is given in Table 1. Of those data, 5743 were used in D for the MQ set and 7007 for the HQ case.

described in paper I, where it was identified as model $\mathrm{B}$, and included a total of 578 variables: coordinates, ADPs, and up to octapole electron population parameters for $\mathrm{C}$ and $\mathrm{O}$ atoms, a single population parameter for the core electrons of these two atomic species, an isotropic extinction coefficient, plus monopole, dipole and quadrupole population coefficients for $\mathrm{H}$ atoms, whose positional parameters were kept fixed at the values obtained in early stages of the refinement. The ADPs of the $\mathrm{H}$ atoms (see Fig. 3) were kept at values calculated by a combination of X-ray and spectroscopic information (Roversi \& Destro, 2004). In view of the close similarities (see below) in $\mathrm{C}$ and $\mathrm{O}$ positional and thermal parameters from the refinement of MQ and HQ data, the same set of atomic ADPs for $\mathrm{H}$ nuclei was employed in the final stages of least-squares refinement of both crystal structures.

Four refinements, A-D, were carried out using the $V A L R A Y$ set of programs (Stewart \& Spackman, 1983): A and B were based on 'no TR corr.' data, C and D on corrected data. Refinements A and $\mathrm{C}$ included all observed $(I>0)$ reflections, while $\mathrm{B}$ and $\mathrm{D}$ were performed on reduced sets,

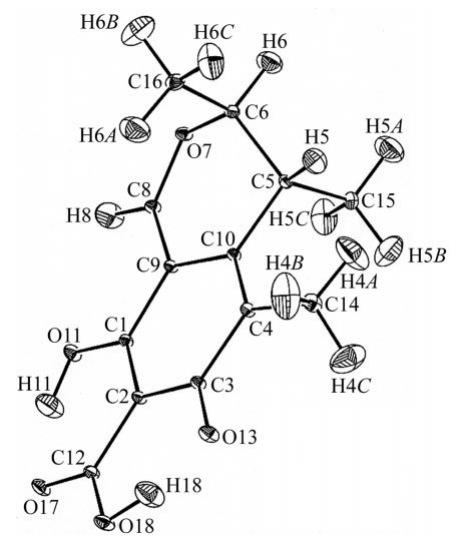

Figure 3

ORTEP plot (Johnson, 1976) of citrinin at $19 \mathrm{~K}$ with the numbering scheme. Ellipsoids at $50 \%$ probability level; those for the $\mathrm{H}$ atoms come from ADPs calculated according to the procedure of Roversi \& Destro (2004). 
Table 4

Differences between the high-quality (HQ) and the medium-quality (MQ) final results for atomic coordinates, bond distances and angles, $U_{\text {eq }}$ 's, $\rho$ and its Laplacian $\nabla^{2} \rho$ at bond (b.c.p.) and ring (r.c.p.) critical points.

Differences are reported as $|\Delta / \sigma|$, with $(A)$ : $\sigma=$ standard uncertainty (s.u.) of the difference $\Delta ;(B): \sigma=$ s.u. from the HQ experiment.

\begin{tabular}{|c|c|c|c|c|}
\hline \multirow[b]{2}{*}{ Property } & \multicolumn{2}{|l|}{$A$} & \multicolumn{2}{|l|}{$B$} \\
\hline & Average & Full range & Average & Full range \\
\hline $\mathrm{C}, \mathrm{O}$ atomic coordinates & 0.7 & $0.0-2.2$ & 2.1 & $0.0-6.3$ \\
\hline $\mathrm{H}$-atom coordinates & 1.0 & $0.0-3.7$ & 2.3 & $0.1-8.9$ \\
\hline Bond distances (no H) $(\AA)$ & 0.7 & $0.1-1.8$ & 2.0 & $0.2-6.0$ \\
\hline Bond angles (no $\mathrm{H})\left({ }^{\circ}\right)$ & 0.6 & $0.0-2.0$ & 1.9 & $0.0-6.5$ \\
\hline $\begin{array}{l}\mathrm{C}-\mathrm{H} \text { and } \mathrm{O}-\mathrm{H} \text { bond } \\
\text { distances }(\AA)\end{array}$ & 1.1 & $0.2-1.9$ & 2.6 & $0.5-4.2$ \\
\hline $\begin{array}{l}\text { H-involving bond } \\
\text { angles }\left({ }^{\circ}\right)\end{array}$ & 0.9 & $0.0-3.8$ & 2.5 & $0.0-9.2$ \\
\hline$U_{\text {eq }}(\mathrm{C}, \mathrm{O}$ atoms $)\left(\AA^{2}\right)$ & 1.4 & $0.1-2.2$ & 3.9 & $0.3-6.4$ \\
\hline $\begin{array}{l}\rho_{\text {b.c.p. }}(\mathrm{C}-\mathrm{C} \text { and } \mathrm{C}-\mathrm{O} \\
\text { bonds })\end{array}$ & 1.9 & $0.0-3.7$ & 5.1 & $0.1-10.2$ \\
\hline $\begin{array}{l}\rho_{\text {b.c.p. }}(\mathrm{C}-\mathrm{H} \text { and } \mathrm{O}-\mathrm{H} \\
\text { bonds })\end{array}$ & 1.0 & $0.2-2.8$ & 3.1 & $0.5-8.9$ \\
\hline$\rho_{\text {r.c.p. }}$ & 2.1 & $1.0-3.3$ & 4.8 & $2.6-7.5$ \\
\hline $\begin{array}{l}\nabla^{2} \rho_{\text {b.c.p. }}(\mathrm{C}-\mathrm{C} \text { and } \mathrm{C}-\mathrm{O} \\
\text { bonds })\end{array}$ & 0.8 & $0.0-1.9$ & 2.4 & $0.0-5.5$ \\
\hline $\begin{array}{l}\nabla^{2} \rho_{\text {b.c.p. }}(\mathrm{C}-\mathrm{H} \text { and } \mathrm{O}-\mathrm{H} \\
\text { bonds })\end{array}$ & 1.0 & $0.0-2.3$ & 2.7 & $0.1-6.2$ \\
\hline$\nabla^{2} \rho_{\text {r.c.p. }}$ & 1.9 & $0.0-3.3$ & 4.2 & $0.0-5.8$ \\
\hline
\end{tabular}

namely only those with $I>2 \sigma(I)$. A summary of the resulting statistical indices is given in Table 3 .

\section{Results and discussion}

The rather large values of $R(F)$ reported in Table 2 for the standard refinement (Sheldrick, 1997) of the RT data sets of the MQ crystal could have been a warning about the adequacy of the sample if citrinin were a regular crystal, not showing disorder at room temperature (see Experimental). Indeed, the first report in the literature on the citrinin crystal structure at room temperature gives an $R$ value of 0.067 (Rodig, 1971) for 919 reflections with $I>3 \sigma(I)$ and measured on a diffractometer equipped with graphite-monochromated $\mathrm{Cu} K \alpha$ radiation. Since we know of this disorder, it has been the profile analysis of the LT data, and not the RT reliability index $R$, that suggested we discard the MQ crystal in the $\rho_{\exp }$ study.

Inspection of Table 3 shows that: (i) on going from A to C, the correction for scan-truncation losses enhances significantly the quality of the fit, owing to its intrinsic improvement of the statistics; (ii) deletion of the weak reflections on going from A to $\mathrm{B}$ (or from $\mathrm{C}$ to $\mathrm{D}$ ) reduces greatly the $R$ values, as expected; (iii) the combination of these two procedures (refinement D) improves the statistical indices to a point that even the MQ case would probably be considered, solely on this basis, a successful and adequate charge-density investigation. Actually, we consider the refinement on all observed $(I>0)$ data, hence refinement $\mathrm{C}$, as the most appropriate basis for the comparison of the MQ and $\mathrm{HQ}$ experiments. Furthermore, in our experience with all-light-atom structures, an indicator of the quality of an X-ray $\rho_{\exp }$ investigation is the
$R(F)$ value on all observed data within the $\mathrm{Cu}$ sphere, which ought to be well below 0.015 in a high-quality experiment regarding a non-centrosymmetric crystal structure. On this ground, Table 3 shows that our citrinin MQ data are not of sufficient quality, whereas the HQ experiment is very satisfactory.

As anticipated by the number of measured reflections and by the $R(F)$ and $w R\left(F^{2}\right)$ values of refinement $\mathrm{C}$ (Table 3), the $\mathrm{HQ}$ data are by far more precise than those of the MQ set. In fact, for all 578 refined parameters the standard uncertainty (s.u.) values of the MQ case are twice to three times as large as those of the HQ set. Obviously, this different precision propagates on all derived quantities.

A first comparison between the outcomes of the HQ and MQ investigations can be made on the conventional structural parameters, i.e. on atomic coordinates, bond distances and angles, and thermal parameters (we have chosen $U_{\text {eq }}$ values). For each quantity, the ratio $|\Delta / \sigma|$ has been evaluated, where $\Delta$ is the difference between the HQ and MQ values and $\sigma$ is the s.u. of $\Delta$. To emphasize the different precision and accuracy of the two experiments, the ratio $|\Delta / \sigma|$ has been computed also with $\sigma$ taken as the s.u. from refinement $\mathrm{C}$ of the HQ data. Table 4 reports the average values of the ratios, as well as the full range of their values. It is seen, in the upper part of the table, that the atomic coordinates from the two crystal structure determinations do not differ, on average, by more than 2.3 HQ s.u., with a largest individual difference (for the $y$ coordinate of atom H5A, see Fig. 3) of 8.9 s.u. Bond distances and angles from the two experiments agree, on average, within $2.6 \mathrm{HQ}$ s.u., with the largest individual
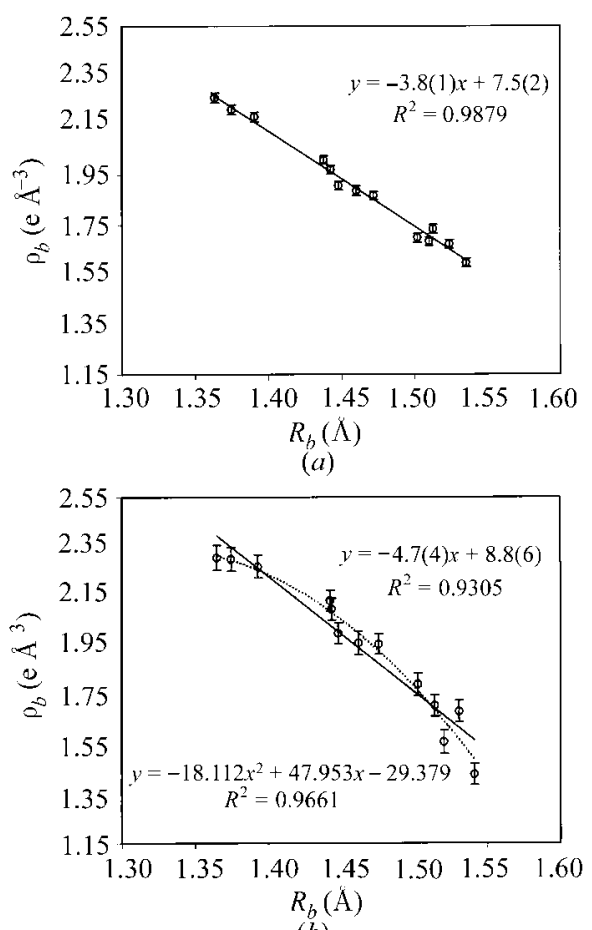

Figure 4

(b)

Values of electron density at the $\mathrm{C}-\mathrm{C}$ bond critical points versus bond path lengths: (a) from the HQ data set, $(b)$ from the MQ data set. 
difference (for the bond angle $\mathrm{C} 5-\mathrm{C} 15-\mathrm{H} 5 A$ ) of 9.2 s.u. Since the $|\Delta / \sigma|$ values for the $U_{\text {eq }}$ 's are also in a rather narrow range, it can be stated that the citrinin molecular structures from MQ and HQ crystals are substantially identical, with possibly significant differences confined to very few quantities.

A second comparison refers to the topological properties of $\rho_{\text {exp }}$. By applying the 'atoms in molecules' theory of Bader (1990), a full search of the bond and ring critical points (c.p.) has been performed with the $P A M o C$ suite of programs (Barzaghi, 2003). For both $\rho_{\text {c.p. }}$ and its Laplacian $\nabla^{2} \rho_{\text {c.p. }}$, differences between the HQ and the MQ electron densities have been evaluated and the ratios $|\Delta / \sigma|$ computed, with $\sigma$ again referring, as before, both to the s.u. of $\Delta$ (set A values in Table 4) and to the s.u. of the HQ values (set B in Table 4). Here too, as noted above for the geometric parameters, the MQ results appear to be in reasonable statistical agreement with the HQ results. However, an overall view of the $B$ values in the table indicates that the actual ratio between the uncertainties of the two sets of results increases when properties mainly related to low-angle data are considered. In particular, for the bond critical points, the number of individual differences between $\rho_{\text {c.p. }}$ values exceeding $6 \mathrm{HQ}$ s.u. is $29 \%$ of the total, much larger than in the case of the geometric parameters. If only $\mathrm{C}-\mathrm{C}$ and $\mathrm{C}-\mathrm{O}$ bonds are considered, the portion of $\Delta \rho_{\text {c.p. }}$ greater than $6 \mathrm{HQ}$ s.u. increases to $37 \%$, thus indicating that the topological details of the electron distribution given by the two experiments are clearly different. Furthermore, a comparison made solely on the basis of $\rho_{\text {c.p. }}$.

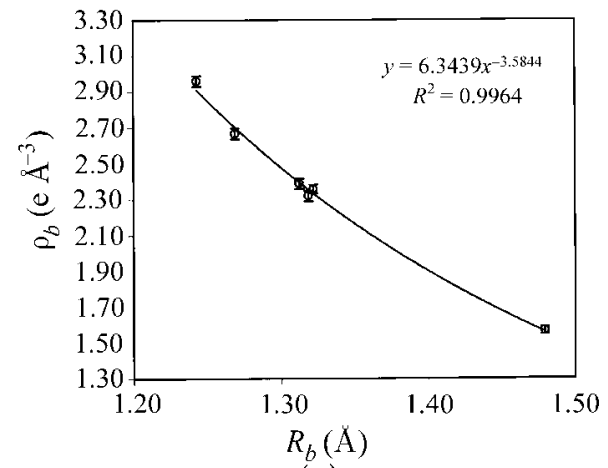

(a)

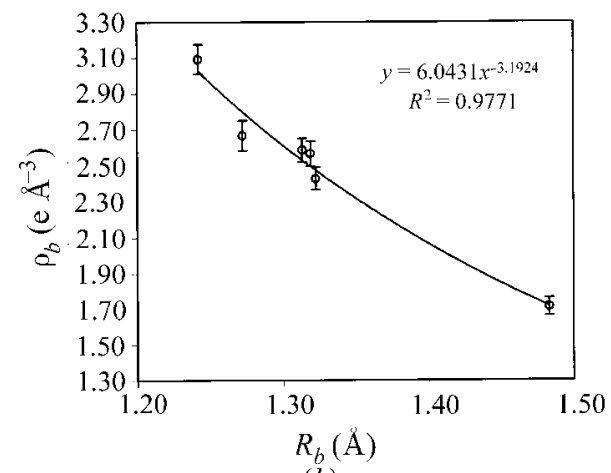

(b)

Figure 5

Same as Fig. 4 for the six $\mathrm{CO}$ bonds and $\nabla^{2} \rho_{\text {c.p. }}$ values might be incomplete. Indeed, in the topological analysis of $\rho_{\exp }$ (especially when comparisons with theoretical charge densities are made), an important role is played by the positions of the critical points, and in the present case many of these differ significantly in the two experimental charge distributions.

A convenient and compact means for judging the quality of the results of a $\rho_{\exp }$ topological analysis is the graphical representation of the correlation between $\rho_{\text {c.p. }}$ values and bond path lengths. Fig. 4(a) reports such quantities for the $\mathrm{C}-\mathrm{C}$ bonds in the $\mathrm{HQ}$ case. It shows that a linear correlation is observed, very similar to that found and discussed in paper I, where the results came from the refinement of a different model against the same HQ data. By contrast, Fig. 4(b) shows that in the MQ case the linear correlation is very poor, to the point that an improbable quadratic function improves considerably the fit (compare the two $R^{2}$ values). Similarly, for the $\mathrm{C}-\mathrm{O}$ bonds, the exponential fit that in paper I was found

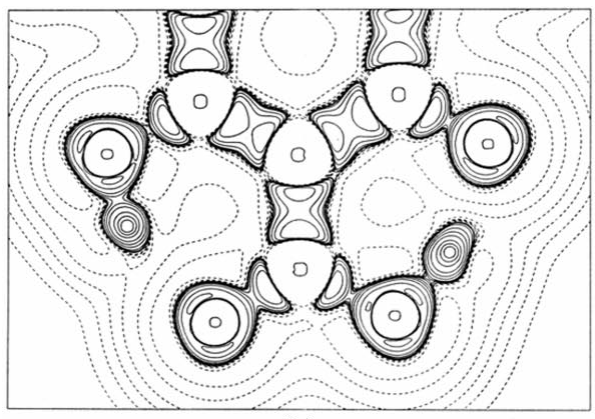

(a)

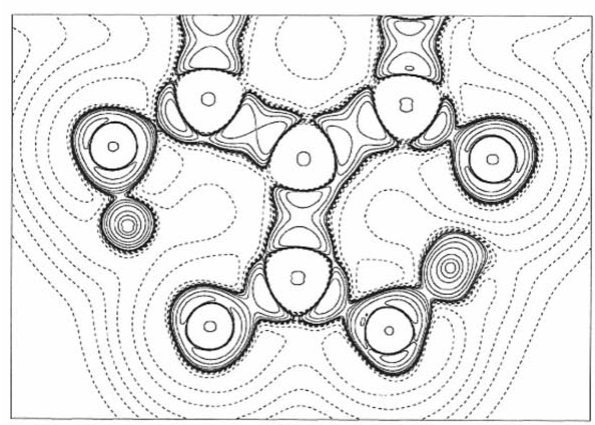

(b)

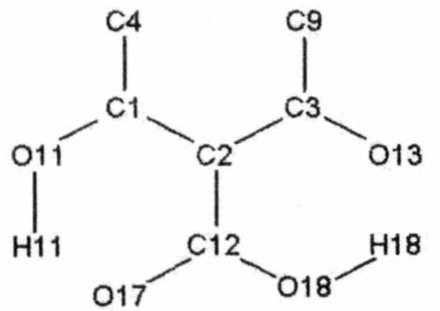

(c)

Figure 6

Contour maps $(7.2 \times 5.0 \AA)$ of the $-\nabla^{2} \rho$ in the plane of rings III and IV of citrinin. Definition of planes and contour levels as in paper I (Roversi et al., 1996). (a) From HQ data; (b) from MQ data; (c) atomic numbering scheme. 


\section{Table 5}

Medium-quality (MQ) and high-quality (HQ) final results for the molecular electric moments of the citrinin molecule.

Units are Debye (D) for the dipole and $\mathrm{e} \AA^{2}$ for the quadrupole moments. The reference system is that of the inertial axes, with the centre of mass as origin.

\begin{tabular}{lcc}
\hline & MQ & HQ \\
\hline Moment components & & \\
First moment & & \\
$\quad \mu_{x}$ & $10(2)$ & $12(1)$ \\
$\mu_{y}$ & $6(1)$ & $7(1)$ \\
$\mu_{z}$ & $-3(1)$ & $-0.4(5)$ \\
Total dipole moment & $12(2)$ & $13(1)$ \\
Second moment & & \\
$\mu_{x x}$ & $-32(1)$ & $-30.3(5)$ \\
$\mu_{x y}$ & $3(1)$ & $2.8(5)$ \\
$\mu_{y y}$ & $-30(1)$ & $-24.9(5)$ \\
$\mu_{x z}$ & $-1(1)$ & $-1.7(5)$ \\
$\mu_{y z}$ & $0.4(6)$ & $0.8(3)$ \\
$\mu_{z z}$ & $-28.3(7)$ & $-28.9(3)$ \\
\hline
\end{tabular}

to be more adequate than the linear fit is well satisfied in the HQ $\rho_{\exp }$ (Fig. $5 a$ ) and worsens markedly in the lower-quality experiment (Fig. 5b).

Another useful way of comparing the $\rho_{\exp }$ from the two investigations is through maps of the Laplacian $\nabla^{2} \rho_{\text {exp }}$, where subtle differences in electron distributions are made more evident. An example is shown in Fig. 6, which reports contour maps of $-\nabla^{2} \rho$ in the plane of rings III and IV of citrinin. It is evident, in the HQ map, that the regions of the two $\mathrm{H}$ atoms, both of which are involved in short intramolecular $\mathrm{O}-\mathrm{H} \cdots \mathrm{O}$ hydrogen bonds, clearly show a charge polarization in the form of $-\nabla^{2} \rho$ peaks elongated towards the contiguous $\mathrm{O}$ atoms at 1.5-1.6 $\AA$. In the MQ map, such a feature is totally absent in the case of atom H11 and vaguely present near atom H18.

A final comparison has been made on the first and second molecular electric moments derived from the analysis of the charge density, and evaluated with PAMoC (Barzaghi, 2003). The results are given in Table 5. The agreement is good, except for the $\mu_{y y}$ component of the quadrupole moment. In particular, the molecular dipole moments are of the same magnitude, within experimental uncertainty, and the angle between the two vectors is slightly less than $13^{\circ}$. Maps of the electrostatic potential in the molecular plane have also been compared and showed a very close similarity. All this suggests that overall molecular properties are confidently obtained even from the MQ set of data.

\section{Conclusions}

The influence of data quality on the results of an experimental charge-density study has been documented for the case of molecular crystals of a relatively small organic compound. A quantitative and detailed discussion of local properties of $\rho_{\exp }$ is meaningful solely when based on data of genuine high quality. If only data of a lower grade are available, the analysis of the electron distribution must be restricted to a qualitative level, while overall molecular properties can still be confidently derived from an adequate multipolar description of $\rho_{\text {exp. }}$. Owing to the possible subjective interpretation of 'lower grade' with respect to 'high quality', the latter statement is not to be interpreted as a suggestion to make use of any kind of diffraction data: what has been reported here as mediumquality data for a crystal tainted by little imperfections was a set of high-resolution X-ray diffracted intensities measured at a temperature as low as $19 \mathrm{~K}$, from a spherical sample, and carefully corrected for scan truncation losses. Very likely, failure to include even one of these conditions in the data collection and processing would have led to further restrictions on the validity of the subsequent charge-density analysis.

\section{References}

Bader, R. F. W. (1990). Atoms in Molecules. A Quantum Theory. New York: Oxford University Press.

Barzaghi, M. (2003). PAMoC, Online User's Manual. CNR-ISTM, Milano, Italy, http://www.istm.cnr.it/ barz/pamoc/.

Coppens, P. \& Becker, P. J. (1995). International Tables for Crystallography, Vol. C, edited by A. J. C. Wilson, p. 627. Dordrecht: Kluwer Academic Publishers.

Destro, R. (1988). Aust. J. Phys. 41, 503-510.

Destro, R. (1991). Chem. Phys. Lett. 181, 232-236.

Destro, R. \& Marsh, R. E. (1984). J. Am. Chem. Soc. 106, 7269-7271.

Destro, R. \& Marsh, R. E. (1987). Acta Cryst. A43, 711-718.

Destro, R. \& Marsh, R. E. (1993). Acta Cryst. A49, 183-190.

Destro, R. \& Merati, F. (1993). Z. Naturforsch. Teil A, 48, 99-104.

Johnson, C. K. (1976). ORTEP-II. Report ORNL-5138, Oak Ridge National Laboratory, Tennessee, USA.

Poupko, R., Luz, Z. \& Destro, R. (1997). J. Phys. Chem. A, 101, 5097-5102.

Rodig, O. R. (1971). Chem. Commun. pp. 1553-1554.

Roversi, P., Barzaghi, M., Merati, F. \& Destro, R. (1996). Can. J. Chem. 74, 1145-1161.

Roversi, P. \& Destro, R. (2004). Chem. Phys. Lett. 386, 472-478.

Sheldrick, G. M. (1997). SHELXL-97. University of Göttingen, Germany.

Sørensen, H. O. \& Larsen, S. (2003). J. Appl. Cryst. 36, 931-939.

Stewart., R. F. (1976). Acta Cryst. A32, 565-574.

Stewart, R. F. \& Spackman, M. A. (1983). VALRAY Users Manual. Carnegie-Mellon University, Pittsburgh, USA. 